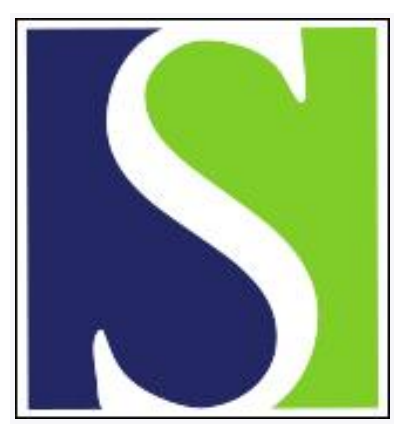

Scand J Work Environ Health 1999;25(6):569-573

https://doi.org/10.5271/sjweh.482

Issue date: Dec 1999

Model development and research vision for the future of multiple chemical sensitivity

by Arnetz BB

The following article refers to this text: 2004;30(6):486-496

Key terms: electric sensitivity; environmental hyperreactivity; environmental illness

This article in PubMed: www.ncbi.nlm.nih.gov/pubmed/10884155

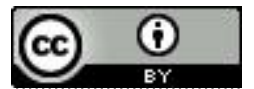




\title{
Model development and research vision for the future of multiple chemical sensitivity
}

\author{
by Bengt B Arnetz, $M D^{\prime}$
}

\begin{abstract}
Arnetz BB. Model development and research vision for the future of multiple chemical sensitivity. Scand J Work Environ Health 1999;25 (6, special issue):569-573.

Multiple chemical sensitivity (MCS) is characterized by heightened self-reported sensitivity to extremely low concentrations of chemicals. It has numerous symptoms in common with the sick building syndrome, the Gulf War syndrome, and chronic fatigue. Despite much research, reproducible objective findings are lacking for MCS, as is a sound model to explain it. This paper proposes a 2-step model combining the needed epidemiologic terminology with that of psychophysiological activation and sensitization. It is suggested that different environmental stressors act as initiators. After initiation, the limbic system and other parts of the brain become sensitized and hyperrreactive to environmental triggers. Odor acts as one important trigger. Future research should use more biological assessments in combination with environmental and psychosocial data and involve patient groups with similar symptoms, although diagnosed as suffering from different entities. The similarities and differences of patients with such entities need to be understood before the entities themselves can be understood, diagnosed, treated, and prevented.
\end{abstract}

Key terms electric sensitivity, environmental hyperreactivity, environmental illness.

Self-reported symptoms and illnesses, for example, chemical sensitivity (CS) and multiple chemical sensitivity (MCS), from exposure to chemicals and odors at supposedly nontoxic levels have initiated an intense debate about the possible cause-and-effect relationships (1, 2). The basis for much of the controversy is that of the biological and toxicologic plausibility.

Proponents of the chemical origin of MCS suggest that both acute exposure to chemicals and long-term repeated exposure to nontoxic levels act as a sensitizer. Sensitization is defined in the neuroscientific and pharmacologic literature as a progressive increase in the response to a set stimulus over time (3). Many traditional toxicologists and occupational health physicians argue that there is a lack of biological and toxicologic plausibility for the notion that nontoxic levels of chemicals would result in symptoms characteristic of MCS. (For a recent review of this debate see reference 2.) Some researchers suggest that MCS and related syndromes may be an expression of psychosomatic disorders, obsessive or paranoid illness, or anxiety or depressive illnesses (46).

One form of MCS is the sick-building syndrome, which, over the years, has attracted substantial research and an array of different types of practical intervention, ranging from replacing building materials to improving ventilation and indoor air quality (7-9). Despite substantial research, we still lack sufficient knowledge to understand fully the truly predictive factors of the sickbuilding syndrome. However, as can be seen in table 1,

Table 1. Symptoms characteristic of multiple chemical sensitivity and the sick-building syndrome.

\begin{tabular}{|c|c|}
\hline Organ & Symptoms \\
\hline Central nervous system & $\begin{array}{l}\text { Headache } \\
\text { Drowsiness, lethargy, malaise } \\
\text { Fatigue } \\
\text { Memory impairment } \\
\text { Difficulty to concentrate } \\
\text { Irritability } \\
\text { Dizziness } \\
\text { Odor and taste complaints }\end{array}$ \\
\hline $\begin{array}{l}\text { Upper respiratory system/ } \\
\text { mucous membrane }\end{array}$ & $\begin{array}{l}\text { Nose symptoms } \\
\text { Throat symptoms, coughs, colds } \\
\text { Dry throat, blocked nose } \\
\text { Eye symptoms, dry eyes, itching eyes }\end{array}$ \\
\hline Lower respiratory system & $\begin{array}{l}\text { Tight chest } \\
\text { Shortness of breath, difficulty to breathe } \\
\text { Wheezing } \\
\text { Flu-like symptoms }\end{array}$ \\
\hline Skin & $\begin{array}{l}\text { Dry,stinging, smarting, itching skin } \\
\text { Reddening, rash }\end{array}$ \\
\hline
\end{tabular}

1 Departments of Public Health and Caring Sciences, Uppsala University and Karolinska Institute.

Reprint requests to: Dr BB Arnetz, Department of Public Health, Uppsala University, Uppsala Science Park, SE-751 85 Uppsala, Sweden. [E-mail: bengt.arnetz@SOCMED.NN.se] 
symptoms characteristic of this syndrome are largely identical to those of MCS. Therefore, future studies need to be more detailed as to specific exposure circumstances precipitating MCS symptoms, as well as patients suffering from the sick-building syndrome. A review of the literature shows that researchers interested in the sickbuilding syndrome predominantly focus on the building, while MCS researchers are more interested in specific chemical exposures, circumstances precipitating illness, and its possible relationship to pathophysiology. Obviously, a more-integrated and multidisciplinary approach is needed to further the understanding of both MCS and the sick-building syndrome. In the future, it would probably benefit both MCS and sick-building-syndrome research to do more integrated studies involving both MCS and sick-building syndrome patients.

More recently, symptoms strikingly similar to those of MCS have been reported among Persian Gulf War veterans $(10,11)$. At least 30000 deployed soldiers of the allied forces have reported symptoms similar to those of MCS following their return to their country of origin. In many cases, the latency period has been several months.

Thus, similar symptoms, such as headache, nausea, memory impairment, memory loss, difficulty to concentrate, dizziness, light-headedness, eye irritation, and fatigue, are common for several related exposure situations in which concern over health effects from chemicals is a common denominator.

In order to enhance our understanding of MCS, researchers need to characterize their research subjects in more detail in terms of socioeconomic background, age, gender, previous exposures, and stimuli eliciting symptoms. Researchers should also provide a detailed description of symptoms. Information on somatic and mental well-being needs to be presented in order to rule out competing reasons for these nonspecific symptoms. The predominance of published reports on MCS lack succinct descriptions of the patients' clinical history, the circumstances surrounding the occurrence of MCS symptoms, and other potential exposures of interest. There are probably both necessary and sufficient factors involved in the initiation of MCS. Research efforts that do not encompass a comprehensive picture of the chemical, physical and psychosocial environments of MCS patients do not add to the existing understanding of MCS and should be discouraged. Mere case descriptions are also unlikely to yield much new scientific knowledge.

Nonspecific symptoms characteristic of MCS are also increasingly reported both in the general occupational health and stress literature (12). Apparently, depending on specific situational and environmental circumstances, patients with similar symptoms tend to attribute them to different causes. The reasons for different attributions may be exposure specifics, individual differences in genetic and biological sensitivity, psychological profiles, and co-factors of potential interest, such as psychophysiological stress and other diseases.

One problem with the current definition of MCS is that it is rather vague and is solely based on self-reports (13). There is a lack of validated and reproducible objective signs. Thus we put a heavy burden on the patients forcing them not only to describe their symptoms, but also to diagnose themselves as suffering from MCS. Since numerous other exposures could result in the same symptoms, it would be a great advantage if we could also find more objective signs of MCS. Some researchers have suggested that there is nasal pathology identifiable by rhinolaryngoscopy (14). However, current knowledge is far from allowing us to diagnose MCS according to inputs other than mere subjective reports and symptoms. This lack of more objective signs also undermines the scientific foundation, and therefore the acceptance of the MCS concept. Nevertheless, the lack of sufficient knowledge does not rule out a unique disease concept due to repeated exposure to chemicals at nontoxic levels.

An integrated model of MCS would be beneficial for future research on this entity. Rather than stating that it is either a pure chemical toxicologic disorder or a psychosomatic disorder, a model is presented allowing the integration of current knowledge about the psychophysiological impact of environmental stressors.

Figure 1 outlines an integrated model for MCS. It is suggested that MCS is the result of a 2-step process. The initial initiation step is characterized by a reset and heightened sensitivity of the limbic system. Researchers typically include the hippocampus, parts of the basal ganglia, the septum area, the amygdala, and the

\begin{tabular}{|c|c|c|}
\hline $\begin{array}{l}\text { Initiation } \\
\text { step } \\
\text { (necessary) }\end{array}$ & $\begin{array}{l}\text { Modifiers } \\
\text { (eg, environment, } \\
\text { genetics) }\end{array}$ & $\begin{array}{l}\text { Hypersensitive/ } \\
\text { reactive limbic } \\
\text { system }\end{array}$ \\
\hline
\end{tabular}

Reversible, no physiologic memory

Figure 1. Integrated 2-step model proposed for multiple chemical sensitivity (MCS). 
hypothalamus, which communicate with all other regions, among limbic structures (15). The limbic system is critically important to the integration of physiological, cognitive, and behavioral responses. If the initial environmental exposure is of sufficient strength, for example, an acute exposure to high levels of chemicals from a spill, to smoke, or to intense psychosocial stress, the impact on the limbic system may be irreversible. Repeated exposure to individually subacute levels of environmental burdens may also result in an initiation process leading to a permanent reset of limbic sensitivity and heightened reactivity. Research during the last decade strongly suggests that repeated exposure to environmental factors of subcritical intensity or acute exposure to intense environmental stressors may actually reset the limbic hypothalmic-pituitary-adrenal (HPA) axis, the limbic-sympathetic axis, and the immunologic system. Subsequently, the resetting of limbic sensitivity will result in major metabolic, autonomic, cardiovascular, and related changes $(16,17)$.

Numerous studies of patients suffering from environmental illness, such as MCS and electric hypersensitivity, report that many subjects exhibit a heightened psychophysiological response to various external stimuli $(18-20)$. Limbic and mesolimbic brain regions are among the most sensitive to repeated, intermittent environmental stimuli. Following the initiation step, there is a progressive and enduring enhancement of the behavioral and neurochemical responses to various environmental stimuli, including low levels of chemicals. It has recently been reported that $1 \mu \mathrm{T}$ of $50-\mathrm{Hz}$ electromagnetic fields impairs sleep, measured by electroencephalography (21). Should these results be reproduced in future research it is possible that electromagnetic fields may act as initiators or as triggers in already initiated patients. Psychological trauma, everyday stressors, and unresolved stress, such as posttraumatic stress disorders, all result in sustained or repeated activation of the limbic system. The important implication of this model for future MCS research is that one needs to consider numerous environmental initiators, not only chemicals but also psychosocial initiators and unresolved stressors from the past. According to the proposed model, any environmental stressor resulting in sustained activation of the limbic system is of relevance to the understanding of why some people develop MCS. The proposed model does not claim that MCS is psychosomatic in origin. It helps explain why certain persons are more susceptible to subtoxic levels of chemicals than others. The arousal results in activation of the hypothalamic-pituitary adrenocortical axis and the sympathetic system. It is suggested that patients with MCS fail to reset their psychophysiological activation to their original level, but rather reach another plateau with higher tension. This phenomenon is described as allostasis - the ability to achieve stability through change (16). Naturally, such factors as individual variations in chemical uptake and phenotypic variations in xenobiotic metabolism may contribute also (22).

After the initiation, subjects exhibit a heightened response to environmental stressors, such as low-level chemical exposures. People can have both a heightened reactivity to chemicals and a sustained reaction. Originally, before the initiation process, the environmental stimulus is not sufficiently potent by itself to evoke any substantial psychophysiological response. Once the organism has been sensitized, it is suggested that symptoms characteristic of environmental illness are elicited at extremely low levels. According to this model, the elicitation occurs at still lower levels should other environmental exposures, such as noise, electromagnetic fields, or mental stressors, coexist with the chemical exposure.

The proposed model differs from Bell's original neuropsychologically based olfactory-limbic model (23). The Bell model suggests that chemicals in the environment gain access to the central nervous system by way of the olfactory and limbic pathways. In the brain, chemicals subsequently cause long-term changes in the limbic system and increase overall cortical arousal. The Bell model predicts that cognitive weaknesses will be associated to a higher degree with the limbic brain regions (ie, the frontal or temporal lobe rather than nonlimbic areas, such as posterior cortex). A recent testing of the Bell hypothesis did not provide any definite conclusion as to the validity of the olfactory-limbic model (24). However, the model proposed here suggests that various stressors, including psychosocial stressors and life trauma, may be sufficient to create measurable changes in the limbic system and increase the sensitivity to environmental triggers, such as odor or noise. The challenge will be to find better objective indicators of such changes in the limbic system. Neurophysiological and neuroendocrine parameters, as well as advanced analyses of the immune system, could provide some opportunity to enhance the ability to measure changes in the reactivity of the central nervous system to environmental stimuli.

This model offers distinctive measurement points, both subjective and more objective ones. Based on the model, research should focus on a better standardization and validation of the instruments used to assess prior exposure. An understanding of prior exposure is critical to an understanding of whether or not an initiation process may have taken place. A prior exposure history should both involve acute toxic exposures and repeated subtoxic exposures. According to the model one needs to consider many more environmental stressors, not only chemicals, of the past. If different latency periods are applied in the analysis, our understanding of the initiation process should provide additional value.

The model also identifies modifying variables, such as current environmental conditions, genetics and 
allostatic load, that is, the resetting of the homeostatic system and its degree of constant adjustment to external and internal challenges.

Since the model suggests that the initiation process results in a limbic system with heightened sensitivity to environmental stimuli, it is possible to carry out various tests of the model. Using already existing exposure models, such as exposure to noise or flickering lights, it would be easy to test specific aspects of the proposed model. Is the response different to exposure to subtoxic levels of specific chemicals, as well as mixtures of chemicals, with and without environmental coexposures? The olfactory system is closely connected with the limbic system, and it is an important warning system to the body. Exposures to multiple odorous chemical inhalants may act as significant warning signals of possible environmental threats (14). It is suggested that the response pattern to environmental odors is influenced by the concurrent activity of the limbic system. In persons with heightened sensitivity and reactivity of the limbic and mesolimbic systems, a set level of chemical exposure will result in a substantially stronger response because the neuroendocrine and neuroimmune systems are set to lengthen short-term survival. The olfactory system, as well as cells in the central and peripheral nervous system, are awash in a milieu interior of stress hormones with a lack of protective anabolic hormones. This phenomenon will subsequently affect the intracellular transcription process and protein synthesis.

I suggest that future research on MCS should address concurrent environmental stressors that trigger the limbic system. This approach is especially important since MCS patients react to environmental concentrations of chemicals at extremely low levels. I suggest that future MCS research models need to apply an integrated physiological model that allows for the impact on such other factors as well. Environmental MCS research will thus increase the understanding of the impact on cellular and subcellular systems of subtoxic chemical exposures and its relevance to subjective and objective signs and disorders. On the basis of existing knowledge, it could strongly be argued that exposure models only encompassing controlled chemical exposures and their effects fail to include critical determinants of the final human response.

In an ongoing study of my group, we are testing subjective and objective effects from exposure to singular chemicals or a mixture of chemicals. By masking certain smells, we are attempting to separate the exposure to a chemical itself from that of the odor effect. We are looking at possible effects from exposure on the nasal mucosa, various stress hormones, such as prolactin and cortisol, but also anabolic hormones (eg, sex hormones). In addition, we are photographing the cornea to study possible exposure-related reddening of the eye. By combining these objective measures with subjective ratings of central nervous system functions, well-being, and specific reactions to various odors, we hope to be able to enhance our understanding of the possible interaction between environmental and internal exposure. Following the more traditional exposure arrangement, we will consider combining numerous environmental stressors with that of low-level chemical exposures. We have tried such combined environmental exposure assessments in studies of patients suffering from electric sensitivity (2).

In conclusion, the vision of future MCS research is that models and experiments to a larger extent apply our current knowledge of how various environmental stressors (chemical, physical and psychosocial) act concurrently to influence the limbic system and its relationship to symptoms characteristic of MCS. Researchers need to reconsider the terminology and consider that similar symptoms, assigned different names, such as MCS, sickbuilding syndrome, and electric sensitivity, could have factors in common, factors whose identification is critical to a better understanding of the etiology and progression of environmental illness. Such knowledge is of paramount importance if the management, treatment and, ultimately, prevention of MCS and related environmental hazards is to be improved.

\section{References}

1. Bell IR, Schwartz GE, Bootzin RR, Wyatt JK. Time-dependent sensitization of heart rate and blood pressure over multiple laboratory sessions in elderly individuals with chemical odor intolerance. Arch Environ Health 1997;52:6-17.

2. Arnetz BB. Environmental illness: multiple chemical sensitivity, sick building syndrome, electric and magnetic filed disease. In: Lundberg A, editor. The Environment and mental health: a guide for clinicians. New Jersey (NY): Lawrence Erlbaum Associates, Publishers, 1998:115-146.

3. Bell IR, Rossi J 3rd, Gilbert ME, Kobal G, Morrow LA, Newlin DB, et al. Testing the neural sensitization and kindling hypothesis for illness from low levels of environmental chemicals. Environ Health Pers 1997;105 suppl 2:539-47.

4. Terr AI. Environmental illness: a clinical review of 50 cases. Arch Intern Med 1986;146:145-9.

5. Rosenberg SJ, Freedman MR, Schmaling KB, Rose C. Personality styles of patients asserting environmental illness. $\mathrm{J}$ Occup Med 1990;32:678-81.

6. Simon GE, Katon WJ, Sparks PJ. Allergic to life: psychological factors in environmental illness. Am J Psychiatry 1990;147:901-6.

7. Menzies R, Tamblyn R, Farant J-P, Hanley J, Nunes F. The effects of varying levels of outdoor-air supply on the symptoms of sick building syndrome. New Engl J Med 1993;328:821-7.

8. Norbäck D, Edling C. Environmental, occupational, and personal factors related to the prevalence of sick building syndrome in the general population. Br J Ind Med 1991;48:45162 
9. Norbäck D, Michel I, Widström J. Indoor air quality and personal factors related to sick building syndrome. Scand J Work Environ Health 1990;16:121-8.

10. National Institutes of Health Technology Assessment Workshop Panel. The Persian Gulf experience and health. JAMA 1994;272:391-6.

11. Kroencke K, Koslowe P, Roy M. Symptoms in 18,495 Persina Guld War veterans: latency of onset and lack of association with self-reported exposures. J Occup Environ Med 1998;40:520-8.

12. Spurgeon A, Gomperz D, Harrington JM. Modifiers of nonspecific symptoms in occupational and environmental syndrome. Occup Environ Med 1996;53:361-6.

13. Cullen MR. The worker with multiple chemical sensitivities: an overview. Occup Med 1987;2:655-66.

14. Meggs WJ, Cleveland, Jr $\mathrm{CH}$. Rhinolaryngoscopic examination of patients with multiple chemical sensitivity syndrome. Arch Environ Health 1993;48:14-8.

15. Nowak R. Windows of the brain: cortisol secretion and depression. J NIH Res 1991:3:62-7.

16. McEwen BS. Protective and damaging effects of stress mediators. N Engl J Med 1998;338:171-9.

17. Sterling $P$, Eyer J. Allostasis: a new paradigm to explain arousal pathology. In: Fisher S, Reason J, editors. Handbook of life stress, cognition and health. New York (NY): John Wiles \& Sons Ltd, 1988;629:49.

18. Bell IR, Schwartz GE, Baldwin CH, Hardin EE. Neural sensitization and physiological markers in multiple chemical sensitivity. Regul Toxicol Pharmacol 1996;24:S39—47.

19. Berg M, Arnetz BB, Lidén S, Eneroth P, Kallner A. Technostress: a psychophysiological study of employees with VDU. associated skin complaints. J Occup Med 1992;34:698-701.

20. Bell IR, Schwartz GE, Bootzin RR, Wyat LK. Time-dependent sensitization of heart rate and blood pressure over multiple laboratory session in elderly individuals with chemical odor intolerance. Arch Environ Health 1997;52:6-17.

21. Åkerstedt T, Arnetz BB, Ficca G, Paulsson L-E, Kallner A. A $50-\mathrm{Hz}$ electromagnetic field impairs sleep. J Sleep Res 1999;8:77-81.

22. McFaden SA. Phenotypic variation in xenobiotic metabolism and adverse environmental response: focus on sulfur-dependent detoxification pathways. Toxicology 1996;111:43-65.

23. Bell I. Possible mechanisms in environmental illness. In: Clinical ecology: a new medical approach to environmental illness. Bolinas (CA): Common Knowledge Press, 1982:32_7

24. Brown-DeGagne A-M, McGlone J. Multiple chemical sensitivity: a test of the olfactory-limbic model. J Occup Environ Med 1999;41:366-77. 